\title{
Time-resolved serial femtosecond crystallography on photoswitchable fluorescent proteins
}

\author{
K. Hadjidemetriou \\ Institut de Biologie Structurale, Univ. Grenoble Alpes, CEA, CNRS, F-38044 Grenoble, France \\ kyprianos.hadjidemetriou@ibs.fr
}

Time-resolved serial femtosecond crystallography (TR-SFX) at X-ray free electron lasers (XFELs) allows studying the structural dynamics of crystalline biological macromolecules down to the sub-picosecond time scale [1]. According to a pump-probe scheme, optical pump pulses initiate activity in light sensitive crystalline proteins and XFEL pulses generate diffraction patterns that allow determining intermediate-state structures. We apply TR-SFX to study light-induced dynamics in a reversibly photoswitchable fluorescent protein, rsEGFP2.

Reversibly photoswitchable fluorescent proteins are essential tools in advanced fluorescence nanoscopy of live cells. They can be repeatedly toggled back and forth between a fluorescent (on) and a non-fluorescent (off) state by irradiation with light at two different wavelengths. Our consortium (*) combines TR-SFX at XFELs, ultrafast absorption spectroscopy and simulation methods to study photoswitching intermediates in rsEGFP2 on the picosecond to nanosecond time scale. We have been able to identify the transient structure of rsEGFP2 in its excited state $1 \mathrm{ps}$ after photoexcitation, and to observe the chromophore in a twisted state, midway between the stable configurations of the on and off states [2]. This observation, together with a ground-state intermediate structure determined $10 \mathrm{~ns}$ after photoexcitation, has allowed us to uncover details of the photo-switching mechanism of rsEGFP2 [3].

Based on the reaction intermediates determined by TR-SFX [2,3] two rationally designed mutants of the rsEGFP2 have been generated. Pico- to nanosecond TR-SFX results experiments on these rsEGFP2 variants have been carried out at SACLA and the LCLS and provide insight into modified energy landscapes (unpublished).

[1] Colletier, J-P., Schirò, G. \& Weik, M. (2018). Time-Resolved Serial Femtosecond Crystallography, Towards Molecular Movies of Biomolecules in Action in X-ray Free Electron Lasers: A Revolution in Structural Biology, edited by Fromme, P., Boutet, S., Hunter. M. Eds., Springer International Publishing, 11:331-356

[2] Coquelle. N., Sliwa. M., Woodhouse. J., Schiro. G., Adam. A. ... Colletier, J-P., I. Schlichting. \& M. Weik. (2018). Nat. Chem. 10, 31-37

[3] Woodhouse. J., ... Sliwa. M., Colletier, J-P., I. Schlichting. \& M. Weik. (2020). Nat. Comm. 11, 1-11

Keywords: $x$-ray free electron lasers; time-resolved studies; photoswitchable fluorescent proteins

(*) the work presented involves a consortium composed of researchers from the Institut de Biologie Structurale, Grenoble, France, Institut Laue Langevin, Grenoble, France, Max-Planck-Institut for Medical Research, Heidelberg, Germany, RIKEN SPring-8 Center, Sayo, Japan, Linac Coherent Light Source, Menlo Park, USA, Max Planck Institute for Biophysical Chemistry, Göttingen, Germany, Laboratoire de Spectrochimie Infrarouge et Raman, Lille, France, Department of Physics, University of Rennes, France, Laboratoire de Chimie-Physique, CNRS/University Paris-Sud, University Paris-Saclay, Orsay, France, Institute of Multidisciplinary Research for Advanced Materials, Tohoku University, Sendai, Japan, Department of Chemistry, Lomonosov Moscow State University, Moscow, Russia, namely, Adam V., Aquila A., Banneville A-S., Barends T., Beschastnov V., Bourgeois D., Boutet S., Byrdin M., Cammarata M., Carbajo S., Chicano T., Christou N., Colletier J-P., Coquelle N., De Zitter E., Demachy I., Doak B., Domratcheva T., El Khatib M., Feliks M., Field M., Fieschi F., Foucar L., Glushonkov O., Gorel A., Grünbein M., Guillon V., Hilpert M., Hunter M., Jakobs S., Jensen N., Joti Y., Kloos M., Koglin J., Lane T., Liang M., Levy B., de la Mora E., Nass-Kovacs G., Owada S., Richard J., Robinson J., Roome C., Ruckebusch C., Schirò G., Schlichting I., Seaberg M., Shoeman R., Sierra R., Sliwa M., Stricker M., Thepaut M., Tono K., Uriarte L., Weik M., Woodhouse J., Yabashi M., You D., Zala N. 